\title{
Modulation of Gamma and Alpha Activity during a Working Memory Task Engaging the Dorsal or Ventral Stream
}

\author{
Daniel Jokisch and Ole Jensen \\ F. C. Donders Centre for Cognitive Neuroimaging, NL-6500 HB Nijmegen, The Netherlands
}

Despite extensive experimental work in both animals and humans, the actual role of oscillatory brain activity for working memory maintenance remains elusive. Gamma band activity $(30-100 \mathrm{~Hz})$ has been hypothesized to reflect either the maintenance of neuronal representations or changing demands in attention. Regarding posterior alpha activity $(8-13 \mathrm{~Hz})$, it is under debate whether it reflects functional inhibition or neuronal processing required for the task. The aim of the present study was to further elucidate the role of oscillatory brain activity in humans using a working memory task engaging either the dorsal or ventral visual stream. We recorded brain activity using magnetoencephalography from subjects performing a delayed-match-to-sample task. Subjects were instructed to remember either the identity or the spatial orientation of shortly presented faces. The analysis revealed stronger alpha power around the parieto-occipital sulcus during retention of face identities (ventral stream) compared with the retention of face orientations (dorsal stream). In contrast, successful retention of face orientations was associated with an increase in gamma power in the occipital lobe relative to the face identity condition. We propose that gamma activity reflects the actual neuronal maintenance of visual representations, whereas the alpha increase is a result of functional inhibition.

Key words: oscillations; working memory; MEG; gamma; alpha; EEG

\section{Introduction}

Working memory is defined as the capacity to retain and manipulate information no longer accessible in the environment (Baddeley, 2003). It has been proposed that oscillatory brain activity plays an essential role for the neuronal dynamics required for sustaining working memory representations (Lisman and Idiart, 1995; Jensen, 2006; Koene and Hasselmo, 2007). Experimentally, oscillations in various frequency bands have been shown to be modulated differently during working memory operations in both humans and animals, but their exact functional role remains to be determined.

Activity in the gamma band has been hypothesized to directly reflect the neuronal correlate of maintained working memory representations. In macaques, sustained gamma activity was measured by intracranial recordings in parietal cortex during a retention period in a visuospatial delayed-match-to-sample task (DMS-task) (Pesaran et al., 2002). In humans, sustained gamma activity during retention of shape information and audiospatial information has been reported in electroencephalography (EEG) and magnetoencephalography (MEG) studies (Tallon-Baudry et al., 1998; Lutzenberger et al., 2002; Kaiser et al., 2003). Intracranial recordings from epileptic patients have revealed a parametric effect in the gamma band with respect to working memory load

Received Dec. 14, 2006; revised Feb. 9, 2007; accepted Feb. 14, 2007.

This work was supported by Volkswagen Foundation Grant I/79876 and Netherlands Organization for Scientific Research Innovative Research Incentive Scheme 864.03.007. We thank Eric Maris, Robert Oostenveld, and Pascal Fries for making the applied analysis tools in the Fieldtrip toolbox available.

Correspondence should be addressed to Dr. Daniel Jokisch, F. C. Donders Centre for Cognitive Neuroimaging, Kapittelweg 29, NL-6525 EN Nijmegen, The Netherlands. E-mail: daniel.jokisch@fcdonders.ru.nl.

DOI:10.1523/JNEUROSCI.5399-06.2007

Copyright $\odot 2007$ Society for Neuroscience $\quad 0270-6474 / 07 / 273244-08 \$ 15.00 / 0$
(Howard et al., 2003; Mainy et al., 2007). Although these studies support the notion that gamma activity plays a role in working memory, it remains unclear whether the observed effects reflect actual maintenance or if they are a consequence of general changes in attention load.

Activity in the alpha band has been found to be strongly modulated in working memory tasks as well. However, its functional role is still under debate. Posterior alpha activity has been proposed to reflect cortical idling (Adrian and Matthews, 1934; Pfurtscheller et al., 1996) or functional inhibition (Klimesch et al., 2007). In the context of working memory operations, it has been found that alpha activity increases parametrically with memory load during retention in the Sternberg task (Jensen et al., 2002; Tuladhar et al., 2007). This finding is consistent with the notion that alpha activity reflects functional inhibition of the dorsal visual stream. Other studies point to a direct computational role of posterior alpha activity. Based on the finding that long-range coherence in the alpha band correlates with perceptual and cross-modal binding, alpha activity has been proposed as a mechanism for controlling interactions between brain regions (Mima et al., 2001; Hummel and Gerloff, 2005).

The aim of the present study was to further elucidate the role of oscillatory activity during working memory maintenance. We chose a working memory task designed to differentially engage the dorsal or the ventral stream. Subjects were required to maintain either the identities or spatial orientations of briefly presented faces. Additionally, we introduced a control task without any working memory demands using the same stimulus material. We hypothesized that if alpha activity reflects inhibition of the dorsal visual stream, alpha power must increase in the dorsal stream during retention of face identities relative to retention of 
face orientations. In contrast, if gamma activity reflects working memory maintenance, an increase in gamma power over dorsal areas is expected when face orientations are maintained.

\section{Materials and Methods}

Participants

Ten healthy volunteers recruited primarily from the Radboud University Nijmegen participated in the study (six males and four females; 23-29 years of age). None of the subjects had a history of neurological disorder. The procedure was approved by the local ethics committee. All subjects had normal or corrected-to-normal vision and provided written consent according to the Declaration of Helsinki.

\section{Procedure and stimuli material}

We used two variants of a DMS-task and one control task, all using images of faces as stimuli. The faces were selected from the database provided by the Max Planck Institute for Biological Cybernetics (Tübingen, Germany) (Troje and Bülthoff, 1996). Faces were presented at 12 different orientations by rotating them $-60^{\circ},-30^{\circ}, 30^{\circ}$, or $60^{\circ}$ according to the vertical axis and $/$ or $-30^{\circ}, 0^{\circ}$, or $30^{\circ}$ according to the ear-to-ear horizontal axis (Fig. 1A). The two variants of the DMS-tasks required the subjects to retain either face identity or orientation. In both versions of the DMS-tasks, subjects had to indicate whether the second sample item matched the probe item either with respect to identity or orientation. The paradigm was complemented by a control task in which subjects had to indicate whether the probe face was rotated to the left or right regardless of the sample face (Fig. $1 B$ ). In the identity and the control task, both the probe and the sample item consisted of a face. In the identity task, each probe face was presented at the same orientation as the sample face to avoid additional task demands from mental rotations of the face representations. In the orientation task, the probe item consisted of a white dot on a black screen, representing the target or a nontarget of the sample face's gaze. Because the gaze of the sample faces was fully determined by the face's orientation, the dot served as an abstract representation of the face's orientation. This procedure was chosen to encourage the subject to retain the face orientation rather than the face identity. Trials were presented in blocks. Each condition consisted of two blocks of 100 trials, resulting in 200 trials per condition. Trials within a block were presented in randomized order. Blocks were administered in counterbalanced order over subjects. Each trial started with an eyeblink command followed by the sample item presented $2 \mathrm{~s}$ later. After a $3 \mathrm{~s}$ delay period, the probe item was presented. Samples and probes were presented for $0.2 \mathrm{~s}$. The likelihood for matching or nonmatching probe items was 50\%. Subjects indicated their responses ("match" or "nonmatch") by button presses using left and right index fingers. The hands used to respectively indicate match and nonmatch responses were counterbalanced over subjects. After each trial, subjects received feedback on their responses ("correct" or "incorrect"). In the control task, subjects had to indicate whether the probe face was oriented to the left or the right by pressing the corresponding button. The sample face did not have any predictive value for the orientation of the probe face (i.e., working memory was not required to solve this task).

\section{$M E G$ recording}

Electromagnetic brain activity was recorded using a whole-head 151 channel axial gradiometer MEG system (Omega 2000; CTF Systems, Port Coquitlam, British Columbia, Canada) installed at the F. C. Donders Centre for Cognitive Neuroimaging (Nijmegen, The Netherlands). The vertical and horizontal electrooculogram (EOG) was recorded as well by bipolar montages. MEG/EOG data were sampled continuously at a rate of $1200 \mathrm{~Hz}$. The subject's head position relative to the MEG sensor array was measured at the beginning and the end of each block by passing current through three coils placed at the cardinal points of the head (nasion and left and right ear canals). For each subject, a full-brain anatomical magnetic resonance imaging (MRI) scan was acquired using a high-resolution inversion-prepared three-dimensional T1-weighted scan sequence using a 1.5 T Siemens Sonata scanner (Siemens, Erlangen, Germany). To allow for precise coregistration of the MEG and MRI data,
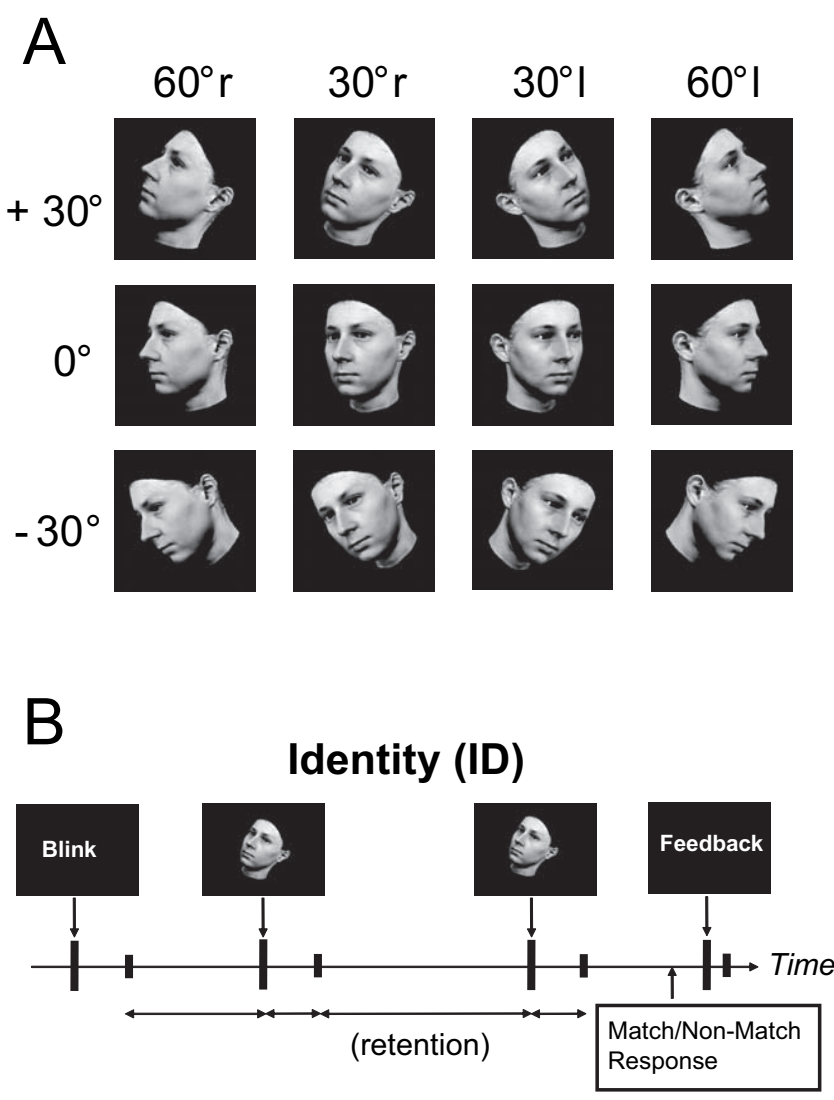

\section{Orientation (OR)}

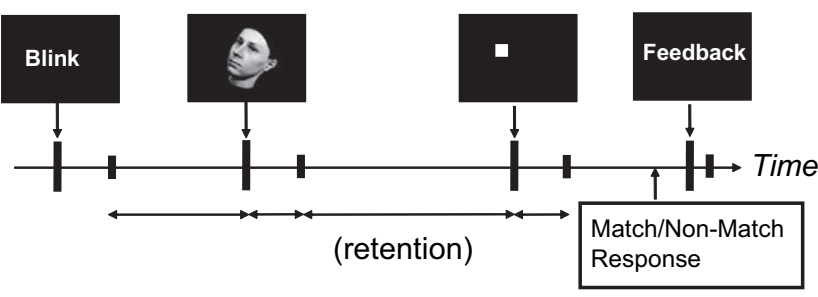

\section{Control (CO)}

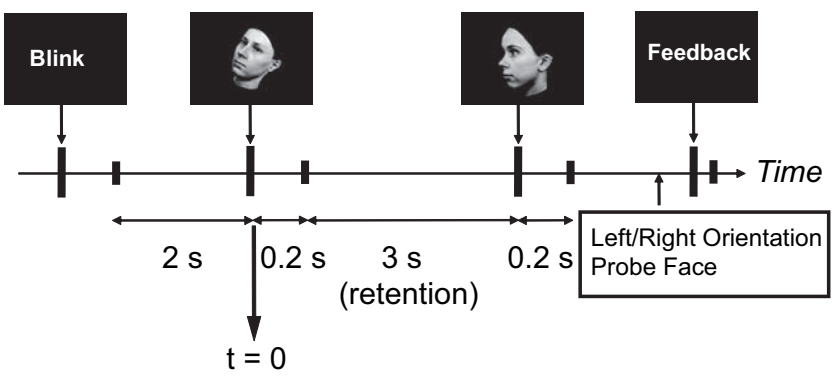

Figure 1. Illustration of stimuli and experimental conditions. $\boldsymbol{A}$, Example set showing the different orientations of the face stimuli. Faces were presented in 12 different orientations (rotated $-60^{\circ},-30^{\circ}, 30^{\circ}$, or $60^{\circ}$ according to the vertical axis and $/$ or $-30^{\circ}, 0^{\circ}$, or $30^{\circ}$ according to the horizontal axis). Faces were presented in color. r, Right; I, left. $\boldsymbol{B}$, Illustration of the three different experimental conditions: DMS-task for face identities (ID), DMS-task for face orientations (OR), and control task with no working memory component $(\mathrm{CO})$. The vertical bars indicate onset and offset of the blink command, the stimuli, and the feedback. In the absence of any command or stimulus, a fixation cross was presented on a black screen. Subjects had to respond to whether the probe item matched the sample with respect to face identity or orientation. In the control task, subjects had to respond whether the second face was oriented toward the right or left regardless of the sample face. 
the left and right ear canals were marked during the scans by two tubes containing vitamin $\mathrm{E}$.

\section{Data analysis}

Data were analyzed using the Fieldtrip software package (http://www. ru.nl/fcdonders/fieldtrip/), a Matlab-based (The MathWorks, Natick, MA) toolbox that has been developed at the F. C. Donders Centre for Cognitive Neuroimaging. Partial artifact rejection was performed by a semiautomatic procedure by rejecting segments of trials containing eyeblinks, muscle artifacts, and jumps in the MEG signal caused by the SQUID (superconducting quantum interference device) electronics. By this procedure, smaller segments of a trial, rather than a whole trial, were rejected. Segments shorter than $0.5 \mathrm{~s}$ were completely rejected to ensure that segments were long enough for subsequent analysis. For the sensorlevel analysis, planar gradients of the MEG field distribution were calculated using a nearest-neighbor method comparable with the method described by Bastiaansen and Knosche (2000); also applied by Osipova et al. (2006). The horizontal and vertical components of the estimated planar gradients approximate the signal measured by MEG systems with planar gradiometers. The planar gradient fields are typically largest in magnitude directly above a given source (Hämäläinen et al., 1993). This is particularly advantageous when interpreting distributions of spectral power at the sensor level.

Spectral analysis. Time-frequency representations of power were calculated for each trial using a multitaper method applied to short sliding time windows (Percival and Walden, 1993). This method allows for controlling the spectral smoothing around the frequency of interest. Two frequency ranges (5-30 and 30-120 Hz) were analyzed separately with different window lengths and smoothing parameters. For the lower frequency band $(<30 \mathrm{~Hz})$, we applied an adaptive time window of five cycles for each frequency $(\Delta T=5 / f)$. By applying three orthogonal Slepian tapers, we achieved an adaptive frequency smoothing of $\Delta f \sim$ $2 / \Delta T$. For the higher frequency range $(>30 \mathrm{~Hz})$, we used a fixed window length of $\Delta T=200 \mathrm{~ms}$. Using three orthogonal Slepian tapers resulted in a spectral smoothing of $\Delta f \sim 10 \mathrm{~Hz}$. In each trial, the power calculated from the Fourier transforms of the tapered time windows were averaged. Power values were separately computed for each sensor location for the horizontal and vertical components of the estimated planar gradients and then summed. Subsequently, the summed planar gradient power estimates were averaged over multiple trials for a given condition. The power values were baseline corrected [relative baseline; $P_{\text {corrected }}(t, f)=$ $\left.\left(P(t, f)-P_{\text {baseline }}(f)\right) / P_{\text {baseline }}(f)\right]$ for the individual frequencies. In the lower frequency range, the baseline was defined from -750 to $-250 \mathrm{~ms}$ with respect to the first stimulus. For the higher frequency range, we used a baseline from -900 to $-100 \mathrm{~ms}$. Thus, these baseline intervals were centered 500 ms before the stimulus.

Statistical analysis. Significant differences between conditions were assessed using a nonparametric randomization test (Nichols and Holmes, 2002; Maris and Oostenveld, unpublished work). This test controls the Type-I error rate with respect to multiple comparisons over sensors using a clustering approach. First, data were averaged over the time and frequency range of interest. Then ordinary $t$-statistics were computed for all sensors. A cluster-finding algorithm identified clusters of contiguous sensors having a threshold below $p<0.05$. Subsequently, the clusterlevel test statistic was defined from the sum of the $t$-values of the sensors in a given cluster. The cluster with the maximum sum was used in the test statistics. The Type I error rate was controlled by evaluating the clusterlevel test statistic under the randomization null distribution of the maximum cluster-level test statistic. This null distribution was computed by randomly reassigning the data to the conditions across multiple subjects and subsequently calculating the test statistics for the new set of clusters. A reference distribution of cluster-level $t$-statistics was created from 1000 random draws. The $p$ value was estimated according to the proportion of the randomization null distribution exceeding the observed cluster-level test statistic. For the comparison of the differences during maintenance of information, we used the interval from 1 to $3 \mathrm{~s}$ after onset of the sample item. This time window was chosen to avoid interference from visual evoked responses. In the statistical analysis, we defined the alpha band from 10 to $11 \mathrm{~Hz}$; however, because of spectral smoothing, the effective
A

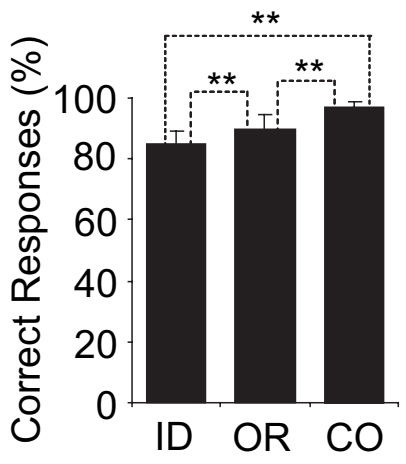

B

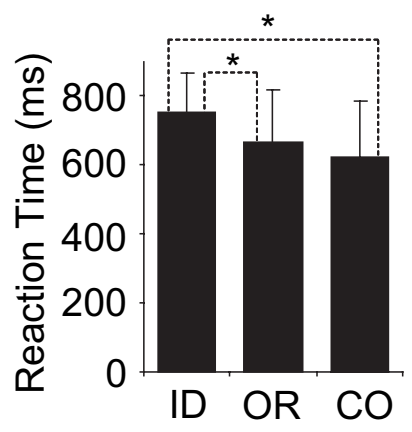

Figure 2. Behavioral data from the three experimental conditions. $\boldsymbol{A}$, Correct responses. $\boldsymbol{B}$, Reaction times. ${ }^{*} p<0.05 ;{ }^{* *} p<0.01$. Error bars indicate $S E M$. The face identity condition was the most demanding. $\mathrm{CO}$, Control; ID, identity; OR, orientation.

frequency band was somewhat broader. For the statistical analysis of the gamma band, we used the frequency range between 70 and $90 \mathrm{~Hz}$. Gamma activity in this frequency range has been found in several studies using similar methods for data analysis to investigate visual perception, spatial working memory, and declarative memory (Hoogenboom et al., 2006; Osipova et al., 2006; Medendorp et al., 2007).

Source analysis. For the identification of the neuronal sources of oscillatory activity, we applied a beamforming approach using an adaptive spatial filtering technique [dynamic imaging of coherent sources (DICS)] (Gross et al., 2001). For source reconstruction, the data from the measured axial sensors (not the planar gradient estimate) were used. The lead field matrices were calculated from anatomically realistic forward head models fitted to the brain surface obtained from each subject's anatomical MRI scan. This was achieved by extending the lead field calculation for a spherical volume conductor by a superposition of basis functions and gradients of harmonic functions constructed from spherical harmonics (Nolte, 2003). The brain of each subject was divided into a grid with a $5 \mathrm{~mm}$ resolution. For each grid location, the lead field was calculated using the forward head model. The DICS approach makes use of the cross- spectral density matrices, which were calculated separately in the baseline and retention intervals. The baseline intervals were chosen as one second before onset of the first stimulus ( -1 to $0 \mathrm{~s}$ ). The retention intervals were required to have the same length as the baseline interval and were chosen to be 1.5-2.5 s after the onset of the first stimulus. Using the cross- spectral density matrices and the lead field matrices, a spatial filter was constructed for each grid point, allowing us to estimate the spatial distribution of power for each condition in each subject. Conditions were compared by subtracting the relative power (power in retention interval divided by power in baseline interval).

The subjects' source reconstructions were overlaid on their individual anatomical MRI scans. Subsequently, the individual anatomical MRI scans and the corresponding power distributions were spatially normalized to the International Consortium for Brain Mapping template (Montreal Neurological Institute, Montreal, Quebec, Canada) by using SPM2 (http://www.fil.ion.ucl.ac.uk/spm). Finally, the source reconstructions were averaged across subjects.

\section{Results}

\section{Behavioral results}

Behavioral performance of correct responses exceeded $85 \%$ across all conditions. Nevertheless, error rates of all three conditions differed significantly (repeated-measure ANOVA; $F_{(2,18)}=$ 44.50; $p<0.001$ ) (Fig. $2 A$ ). Most errors were done in the face identity condition. Reaction times were significantly different between the conditions $\left(F_{(2,18)}=8.59 ; p=0.002\right)$ (Fig. $2 B$ ). For correct responses, reaction times were significantly longer for the face identity compared with the control condition, whereas they 
A
ID

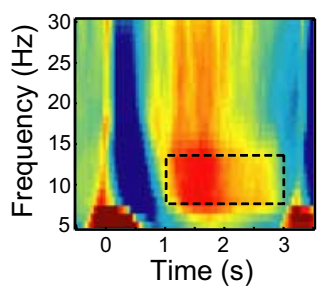

B

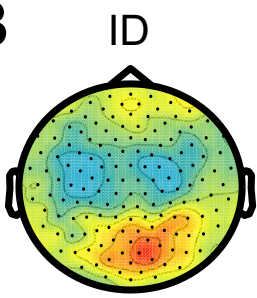

C

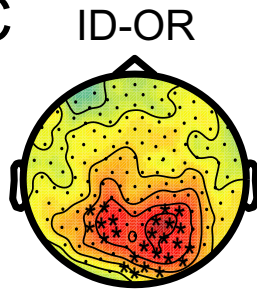

OR

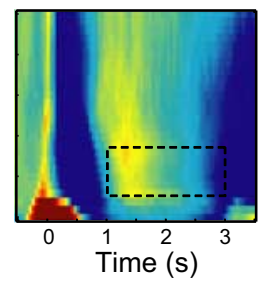

Relative Power
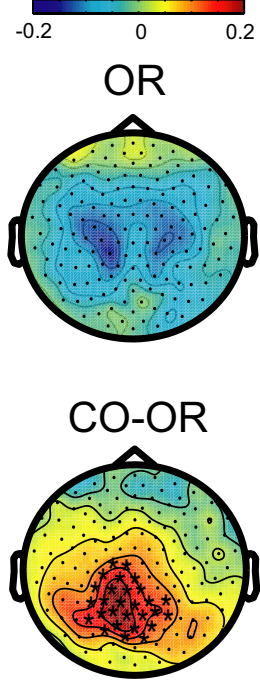

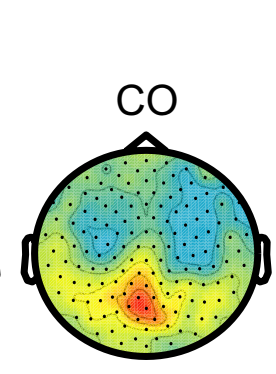

$\mathrm{CO}$


Figure 3. The alpha band activity during retention. $A$, Grand average of the TFRs of the posterior activity from the mean of parieto-occipital sensors (ML011, ML012, ML021, ML032, MLP31, MR011, MR012, MR021, MRP31, and MR032). The sample item was presented at $t=$ $0 \mathrm{~s}$, and the probe item was presented at $t=3.2 \mathrm{~s}$. A relative prestimulus baseline was applied. The boxes (dashed lines) indicate the time-frequency interval for which topographies were calculated. $\boldsymbol{B}$, Grand average of the topography of alpha power $(8-13 \mathrm{~Hz})$ during the retention interval (1-3s). C, Differential topographical plots of alpha power during the retention interval $(1-3 \mathrm{~s})$ for the different conditions compared. Asterisks denote clusters of significant differences $(p<0.05)$. CO, Control; ID, identity; OR, orientation.

were not significantly different for the face orientation compared with the control condition. In short, the behavioral measures showed that the face identity task was the most demanding condition, and the control task was the easiest.

\section{Modulations in the alpha band}

Time-frequency representations of power (TFRs) for the combined planar gradients were calculated for the three different experimental conditions (Fig. $3 A, B)$. In the alpha band $(8-13 \mathrm{~Hz}$ ), we observed an increase in activity over posterior areas during the interval between the sample and the probe stimulus in the face identity and in the control condition. In contrast, alpha activity did not increase in the face orientation condition during the retention interval. To assess the significance of differences in the retention interval between the conditions, a nonparametric randomization test controlling for multiple comparisons over sensors was applied (see Materials and Methods). Then, spectral power was averaged over frequency $(10-11 \mathrm{~Hz})$ and the retention interval (1-3 s). We excluded the first second of the retention interval because this period contains evoked responses as well. When comparing the face identity and orientation conditions in the alpha band, the randomization routine identified two signif-

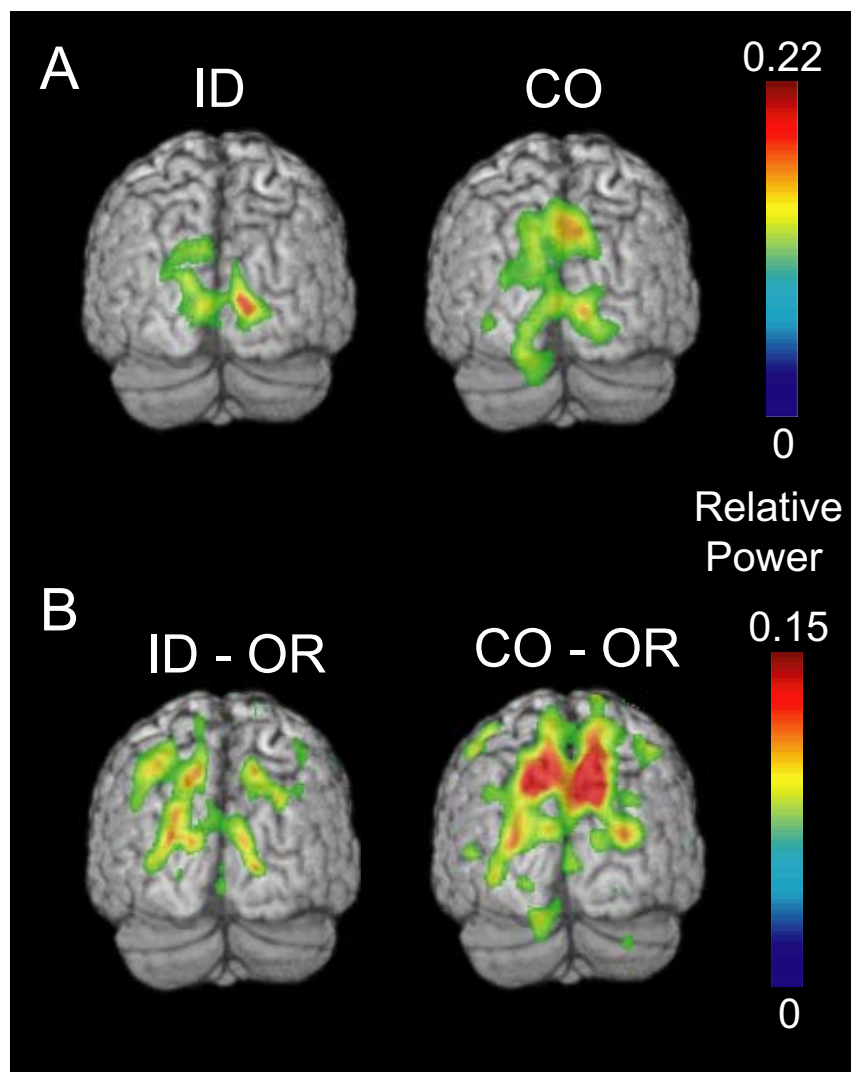

Figure 4. Source analysis of the alpha power during the retention interval. $\boldsymbol{A}$, The identity (ID) and control (CO) condition compared with the prestimulus baseline showed alpha sources in the occipital lobe extending up to the parieto-occipital sulcus. No reliable sources were identified for the orientation (OR) condition. $\boldsymbol{B}$, Comparisons for which the sensor-level analysis revealed significant differences (ID-OR and $C O-O R$ ). The sources concentrated around the parieto-occipital sulcus. Source reconstructions were calculated according to the anatomy of each subject, normalized to a standard brain, and averaged. The overlaid power values were thresholded at $60 \%$ of the maximum.

icant clusters of parieto-occipital sensor $(p<0.05)$ (Fig. $3 C)$. The comparison between the control condition and the face orientation condition revealed one large significant cluster $(p<0.05)$. Finally, the face identity and the control condition were compared, but no significant clusters were identified.

To further characterize the oscillatory alpha activity, we applied a beamforming approach to identify the corresponding sources. As shown in Figure 4, alpha sources were located around the parieto-occipital sulcus. The location of these sources is consistent with the topography of power at the sensor level as represented in Figure 3C. Comparing differences in source strength for retention of face identities versus retention of spatial orientations revealed occipital and parietal sources. Comparing differences in source strength for the control condition versus the orientation condition revealed to a larger extent sources around the parietooccipital sulcus.

\section{Modulations in the gamma band}

Subsequently, we applied the TFRs to characterize modulations in gamma band activity. Figure $5 A$ illustrates strong gamma power $(70-90 \mathrm{~Hz})$ during the presentation of the sample $(t=$ $0-0.2 \mathrm{~s})$ and probe items $(t=3.2-3.4 \mathrm{~s})$ for all three conditions. In the orientation condition, we observed sustained gamma power in the retention interval. As seen in Figure $5 B$, the gamma 

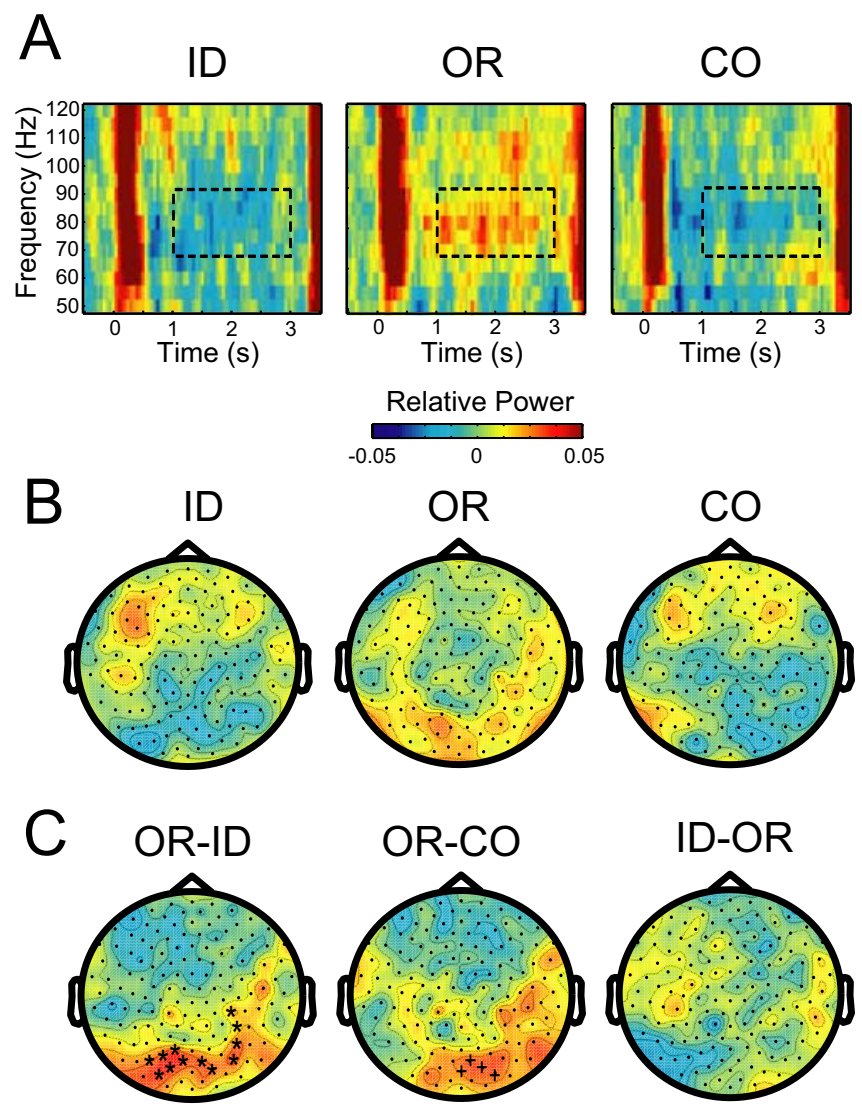

Figure 5. The gamma band activity during retention. $\boldsymbol{A}$, Grand average of the TFRs of the posterior activity from the mean of parieto-occipital sensors (as in Fig. 3). The sample item was presented at $t=0 \mathrm{~s}$, and the probe item was presented at $t=3.2 \mathrm{~s}$. The boxes (dashed lines) indicate the time-frequency interval for which topographies were calculated. $\boldsymbol{B}$, Grand average of the topography of gamma power $(70-90 \mathrm{~Hz})$ during the retention interval (1-3 s). C, Differential topographical plots of gamma power during the retention interval (1-3s) for the different conditions compared. Asterisks $(p<0.05)$ and plus signs $(p<0.10)$ denote clusters of significant differences and clusters showing a tendency toward a significant difference, respectively. CO, Control; ID, identity; OR, orientation.

power for the orientation condition during retention was mainly posteriorly distributed. However, an indication of a left frontal gamma source was also observed in all of the conditions. Statistical analysis using the cluster randomization test revealed a significant cluster of occipital sensors for the comparison between face orientations and face identities in the gamma band $(p<$ 0.01 ) (Fig. 5C). The comparison between face orientations and the control conditions showed a trend toward a significant difference in the gamma band for a cluster of occipital sensors ( $p=$ $0.073)$. Other studies have reported working memory effects in the lower gamma band $(30-60 \mathrm{~Hz})$ (Tallon-Baudry et al., 1998); however, the effects we observed were constrained to the higher gamma band.

The beamforming technique enabled us to localize the gamma activity measured during retention of face orientations. As seen in Figure 6, the sources were strongest in the occipital lobe. When comparing the retention of face identity to the retention of face orientation, sources in the occipital lobe were identified again. There was also an indication of a source in the right cerebellum. However, given the relative weak signal-to-noise ratio of the gamma power, these results should be interpreted with caution. Nevertheless, the gamma sources in the occipital lobe appear

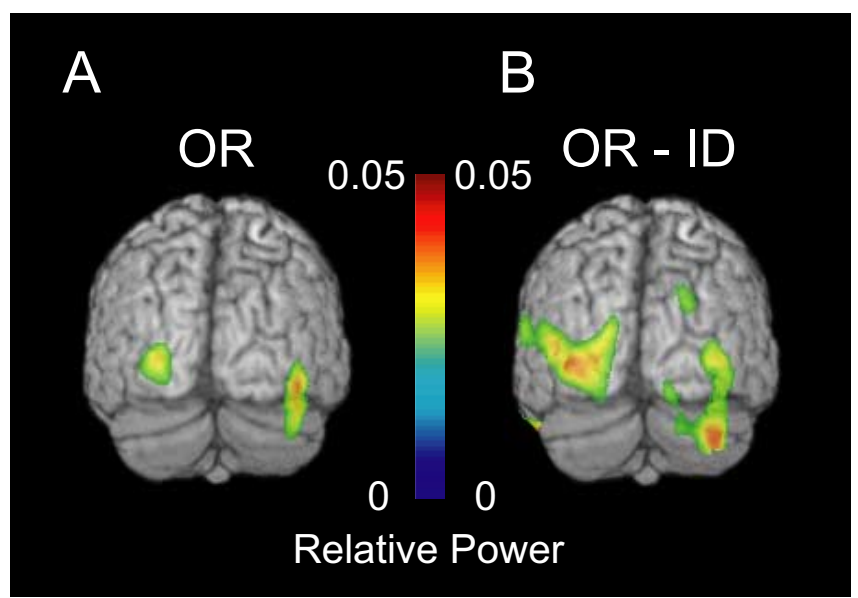

Figure 6. Source analysis of the gamma power during the retention interval. $\boldsymbol{A}$, The orientation condition (OR) compared with the prestimulus baseline revealed gamma sources in the occipital lobe. No reliable sources were identified in the identity or control condition. B, Comparisons for which the sensor-level analysis revealed significant differences (OR-ID). The sources were in the occipital lobe. The values were thresholded at $60 \%$ of the maximum power values.

consistently and are in accord with the topography represented in Figure $5 C$. Although we identify the gamma sources in occipital areas, we cannot exclude that more anterior sources in parietal cortex are engaged as well (Pesaran et al., 2002). For instance, field cancellation caused by differently oriented sources could attenuate parietal gamma activity.

Given that a block design was applied, one might be concerned that systematic effects in the baseline interval interfered with the modulations in the retention interval. We subjected the data in the alpha and gamma band in the baseline intervals to the cluster-randomization statistical analysis to compare the conditions. In the posterior sensors in which the effects were identified between the conditions, we did not find significant power differences in the baseline interval. Additionally, it was during the orientation condition that posterior gamma was the strongest and posterior alpha was the weakest. According to the behavioral measures, the identity and the control condition were respectively more difficult and easier than the orientation condition. We conclude that systematic changes in difficulty resulting in changes in the baselines cannot account for the reported alpha and gamma effects.

\section{Discussion}

In the current study, we investigated the role of oscillatory activity during working memory maintenance. We applied a DMS-task in which the subjects had to maintain either the identity or orientation of visually presented face stimuli. Maintenance of face identities resulted in increases of posterior alpha activity generated around the parieto-occipital sulcus. Maintenance of face orientations resulted in attenuated alpha activity, whereas posterior gamma power increased. The sources of the gamma power were localized in the occipital lobe. Given that the behavioral results showed that the face identity task was more difficult than the orientation task, the increase in gamma was explained by memory retention rather than increases in task demands.

Gamma activity reflects working memory maintenance rather than attention

The working memory task that we applied was specifically designed to engage the dorsal or ventral visual stream by asking 
subjects to retain face identities or face orientations, respectively. In the face orientation task, presumably engaging the dorsal stream, we found clear evidence of sustained gamma activity extending the full retention period (Fig. $5 A$ ). The gamma activity was not present in the face identity task, although this was the most demanding condition, as revealed by error rates and reaction times (Fig. 2). Thus, the sustained gamma activity cannot be explained by an increase in nonspecific attention load. This is different from previous human EEG and MEG studies reporting increased gamma activity during working memory retention compared with a simple control task (Tallon-Baudry et al., 1998; Kaiser et al., 2003). We propose that the gamma activity directly reflects sustained firing of neuronal representations of the spatial memory representations. The sustained activation in early visual areas is compatible with the idea of a visuospatial sketch pad maintaining the spatial representations (Baddeley, 1998).

Why was gamma activity not observed during maintenance in the face identity task? The orientation task is spatial in nature, and the working memory system might make use of the topographic organization in early visual areas. Maintenance of faces, however, is likely to rely on higher-order areas like the fusiform face area (FFA) (Druzgal and D'Esposito, 2003; Postle et al., 2003; Ranganath et al., 2004a,b). Thus, one might have expected gamma activity over ventral areas, but we did not observe this. The absence of ventral gamma activity might be explained by the FFA being relatively distant with respect to the MEG sensors, thus producing poorly detectable signals.

The idea of maintaining working memory representations by sustained firing dates back to Hebb (1949). However, consistent with other studies, we find that the sustained activity is rhythmically synchronized in the gamma band (Tallon-Baudry et al., 1998; Lutzenberger et al., 2002; Pesaran et al., 2002; Medendorp et al., 2007). What is the functional role of the sustained activity being modulated by gamma synchronization? Experimental and theoretical work shows that a neuron receiving multiple inputs is more likely to fire if these inputs are synchronized (for review, see Salinas and Sejnowski, 2001). Thus, the synchronized neuronal gamma activity in the occipital lobe during retention is likely to result in a stronger drive to areas further processing the spatial working memory representations. This processing might include manipulation of the representations or long-term memory encoding (Osipova et al., 2006).

\section{Alpha activity reflects functional inhibition}

We observed a clear increase in alpha power when face identities but not face orientations were maintained. Because the set of sample stimuli were identical in all conditions and only instructions were varied, rebound effects after visual stimulation cannot account for the differential modulations in alpha activity. Given that maintenance of face identities is more demanding than face orientations (Fig. 2), one might suggest that the increase in alpha power is explained by an increase in memory load as observed in the Sternberg task (Jensen et al., 2002; Tuladhar et al., 2007). This interpretation is contradicted by our findings demonstrating that alpha power is also strong in the control task, which is the least demanding condition. Thus, a more likely interpretation is that the increase in alpha power reflects the inhibition of the dorsal stream during the maintenance of face identities, which engages the ventral stream. During maintenance of face orientations, the dorsal stream is required, resulting in attenuated alpha power. Increased dorsal alpha power in the control task can be explained by the fact that the representation of the orientation of the first face is suppressed to avoid interference with the second face's orientation, which is crucial to solve the control task. In conclusion, our findings provide additional support for the alpha inhibition hypothesis (Jensen et al., 2002; Klimesch et al., 2007). According to this idea, alpha activity is a consequence of a top-down control of posterior brain areas (von Stein et al., 2000).

Alternatively, one might argue that alpha reflects a general suppression mechanism not only restricted to the dorsal stream. According to this scheme, alpha reflects suppression of input during retention in all three tasks. Alpha activity in the orientation task is weaker than in the face identity task because this task is easier, as shown by the behavioral data. In the control task, alpha reflects the suppression of the first item to avoid interference with the second item being important for the task.

Additional support for the notion that alpha activity reflects active functional inhibition comes from studies on visuospatial attention and working memory (Worden et al., 2000; Kelly et al., 2006; Thut et al., 2006; Medendorp et al., 2007). These studies demonstrate that attention directed toward one visual hemifield is expressed as a contralateral decrease and/or ipsilateral increase of posterior alpha power.

Why did we not observe an alpha power increase over the ventral stream in the spatial working memory task? One explanation is that the ventral stream, and in particular the FFA, is farther away from the sensors than the dorsal stream, thus producing a smaller signal. However, given the typically strong magnitude of the alpha activity, a more likely explanation is that alpha activity is only produced by the dorsal but not the ventral stream. This is consistent with EEG/MEG studies localizing the alpha source in the dorsal but not the ventral stream (Hari and Salmelin, 1997).

\section{Relationship with other imaging modalities}

Other imaging techniques like functional MRI (fMRI) have been applied to study memory maintenance of faces (Courtney et al., 1997; Druzgal and D’Esposito, 2003; Postle et al., 2003; Ranganath et al., 2004a,b) and spatial representations (Awh et al., 1999; Postle and D'Esposito, 1999; Zarahn et al., 1999; Postle et al., 2000, 2004; Leung et al., 2002; Beurze et al., 2007; Postle, 2006). These studies primarily focus on the role of prefrontal and posterior areas in working memory operations. Nevertheless, consistent with our results, several of these studies do report sustained activity in the occipital lobe when spatial information is maintained (Awh et al., 1999; Postle and D'Esposito, 1999; Postle et al., 2004; Postle, 2006). Although numerous fMRI studies have reported that prefrontal areas are engaged in working memory maintenance (Passingham and Sakai, 2004; Ranganath and D'Esposito, 2005; Curtis, 2006), we did not find robust MEG signals over prefrontal areas being modulated by the different conditions. These differences are most likely explained by the differences in sensitivity to neuronal activity measured by the blood oxygenation level-dependent (BOLD) signal and by electrophysiological measures. For instance, multiple frontal sources might be cancelled out if the sources are not well aligned. We conclude that in the context of working memory tasks, fMRI and MEG provide complementary measures of neuronal activity.

\section{Alpha and gamma activity were inversely related}

Increases in alpha and gamma power were inversely related. This finding is consistent with the notion that gamma activity reflects active neuronal computation, whereas alpha activity reflects functional inhibition. Several imaging studies have shown that power in the gamma band correlates positively with the BOLD response (Logothetis et al., 2001; Moosmann et al., 2003; Mukamel et al., 2005; Niessing et al., 2005), whereas power in the alpha 
band correlates negatively (Goldman et al., 2002; Laufs et al., 2003; Moosmann et al., 2003; Kilner et al., 2005). Thus, we propose that the BOLD changes observed in visual areas in spatial working memory tasks (Awh et al., 1999; Postle and D'Esposito, 1999; Postle et al., 2004; Postle, 2006) are a consequence of oscillatory activity shifting from the alpha to the gamma band. Whether primarily modulations in gamma or alpha activity result in the BOLD changes remains to be determined.

In this study, we found support for the notion that posterior $\alpha$ activity is related to functional inhibition of the dorsal stream when the ventral stream is engaged in working memory maintenance. Furthermore, we demonstrated that posterior gamma activity in the occipital lobe is related to working memory maintenance of spatial representations rather than global changes in attention. This opens the exciting possibility that the early visual system is partly responsible for maintaining the working memory trace of spatial representations. Future studies (e.g., using transcranial magnetic stimulation) are required to establish to what extent the occipital region is required for successful working memory maintenance of spatial representations.

\section{References}

Adrian ED, Matthews BH (1934) The Berger rhythm: potential changes from the occipital lobes in man. Brain 57:355-385.

Awh E, Jonides J, Smith EE, Buxton RB, Frank LR, Love T, Wong EC, Gmeindl L (1999) Rehearsal in spatial working memory: evidence from neuroimaging. Psychol Sci 10:433-437.

Baddeley A (2003) Working memory: looking back and looking forward. Nat Rev Neurosci 4:829-839.

Baddeley AD (1998) Human memory: theory and practice, revised edition. Boston: Allyn and Bacon.

Bastiaansen MC, Knosche TR (2000) Tangential derivative mapping of axial MEG applied to event-related desynchronization research. Clin Neurophysiol 111:1300-1305.

Beurze SM, De Lange FP, Toni I, Medendorp WP (2007) Integration of target and effector information in the human brain during reach planning. J Neurophysiol 97:188-199.

Courtney SM, Ungerleider LG, Keil K, Haxby JV (1997) Transient and sustained activity in a distributed neural system for human working memory. Nature 386:608-611.

Curtis CE (2006) Prefrontal and parietal contributions to spatial working memory. Neuroscience 139:173-180.

Druzgal TJ, D’Esposito M (2003) Dissecting contributions of prefrontal cortex and fusiform face area to face working memory. J Cogn Neurosci 15:771-784.

Goldman RI, Stern JM, Engel Jr J, Cohen MS (2002) Simultaneous EEG and fMRI of the alpha rhythm. NeuroReport 13:2487-2492.

Gross J, Kujala J, Hamalainen M, Timmermann L, Schnitzler A, Salmelin R (2001) Dynamic imaging of coherent sources: studying neural interactions in the human brain. Proc Natl Acad Sci USA 98:694-699.

Hämäläinen M, Hari R, Ilmoniemi RJ, Knuutila J, Lounasmaa OV (1993) Magnetoencephalography - theory, instrumentation, and applications to noninvasive studies of the working human brain. Rev Mod Phys 65:413-497.

Hari R, Salmelin R (1997) Human cortical oscillations: a neuromagnetic view through the skull. Trends Neurosci 20:44-49.

Hebb DO (1949) The organization of behavior; a neuropsychological theory. New York: Wiley.

Hoogenboom N, Schoffelen JM, Oostenveld R, Parkes LM, Fries P (2006) Localizing human visual gamma-band activity in frequency, time and space. NeuroImage 29:764-773.

Howard MW, Rizzuto DS, Caplan JB, Madsen JR, Lisman J, AschenbrennerScheibe R, Schulze-Bonhage A, Kahana MJ (2003) Gamma oscillations correlate with working memory load in humans. Cereb Cortex 13:1369-1374.

Hummel F, Gerloff C (2005) Larger interregional synchrony is associated with greater behavioral success in a complex sensory integration task in humans. Cereb Cortex 15:670-678.

Jensen O (2006) Maintenance of multiple working memory items by temporal segmentation. Neuroscience 139:237-249.
Jensen O, Gelfand J, Kounios J, Lisman JE (2002) Oscillations in the alpha band $(9-12 \mathrm{~Hz})$ increase with memory load during retention in a shortterm memory task. Cereb Cortex 12:877-882.

Kaiser J, Ripper B, Birbaumer N, Lutzenberger W (2003) Dynamics of gamma-band activity in human magnetoencephalogram during auditory pattern working memory. NeuroImage 20:816-827.

Kelly SP, Lalor EC, Reilly RB, Foxe JJ (2006) Increases in alpha oscillatory power reflect an active retinotopic mechanism for distracter suppression during sustained visuospatial attention. J Neurophysiol 95:3844-3851.

Kilner JM, Mattout J, Henson R, Friston KJ (2005) Hemodynamic correlates of EEG: a heuristic. NeuroImage 28:280-286.

Klimesch W, Sauseng P, Hanslmayr S (2007) EEG alpha oscillations: the inhibition-timing hypothesis. Brain Res Brain Res Rev 53:63-88.

Koene RA, Hasselmo ME (2007) First-in-first-out item replacement in a model of short-term memory based on persistent spiking. Cereb Cortex, in press.

Laufs H, Kleinschmidt A, Beyerle A, Eger E, Salek-Haddadi A, Preibisch C, Krakow K (2003) EEG-correlated fMRI of human alpha activity. NeuroImage 19:1463-1476.

Leung HC, Gore JC, Goldman-Rakic PS (2002) Sustained mnemonic response in the human middle frontal gyrus during on-line storage of spatial memoranda. J Cogn Neurosci 14:659-671.

Lisman JE, Idiart MA (1995) Storage of $7+/-2$ short-term memories in oscillatory subcycles. Science 267:1512-1515.

Logothetis NK, Pauls J, Augath M, Trinath T, Oeltermann A (2001) Neurophysiological investigation of the basis of the fMRI signal. Nature 412:150-157.

Lutzenberger W, Ripper B, Busse L, Birbaumer N, Kaiser J (2002) Dynamics of gamma-band activity during an audiospatial working memory task in humans. J Neurosci 22:5630-5638.

Mainy N, Kahane P, Minotti L, Hoffmann D, Bertrand O, Lachaux JP (2007) Neural correlates of consolidation in working memory. Hum Brain Mapp 28:183-193.

Medendorp WP, Kramer GFI, Jensen O, Oostenveld R, Schoffelen JM, Fries P (2007) Oscillatory activity in human parietal and occipital cortex shows hemispheric lateralization and memory effects. Cereb Cortex, in press.

Mima T, Oluwatimilehin T, Hiraoka T, Hallett M (2001) Transient interhemispheric neuronal synchrony correlates with object recognition. J Neurosci 21:3942-3948.

Moosmann M, Ritter P, Krastel I, Brink A, Thees S, Blankenburg F, Taskin B, Obrig H, Villringer A (2003) Correlates of alpha rhythm in functional magnetic resonance imaging and near infrared spectroscopy. NeuroImage 20:145-158.

Mukamel R, Gelbard H, Arieli A, Hasson U, Fried I, Malach R (2005) Coupling between neuronal firing, field potentials, and FMRI in human auditory cortex. Science 309:951-954.

Nichols TE, Holmes AP (2002) Nonparametric permutation tests for functional neuroimaging: a primer with examples. Hum Brain Mapp 15:1-25.

Niessing J, Ebisch B, Schmidt KE, Niessing M, Singer W, Galuske RA (2005) Hemodynamic signals correlate tightly with synchronized gamma oscillations. Science 309:948-951.

Nolte G (2003) The magnetic lead field theorem in the quasi-static approximation and its use for magnetoencephalography forward calculation in realistic volume conductors. Phys Med Biol 48:3637-3652.

Osipova D, Takashima A, Oostenveld R, Fernandez G, Maris E, Jensen O (2006) Theta and gamma oscillations predict encoding and retrieval of declarative memory. J Neurosci 26:7523-7531.

Passingham D, Sakai K (2004) The prefrontal cortex and working memory: physiology and brain imaging. Curr Opin Neurobiol 14:163-168.

Percival DB, Walden AT (1993) Spectral analysis for physical applications: multitaper and conventional univariate techniques. Cambridge, UK: Cambridge UP.

Pesaran B, Pezaris JS, Sahani M, Mitra PP, Andersen RA (2002) Temporal structure in neuronal activity during working memory in macaque parietal cortex. Nat Neurosci 5:805-811.

Pfurtscheller G, Stancak Jr A, Neuper C (1996) Event-related synchronization (ERS) in the alpha band-an electrophysiological correlate of cortical idling: a review. Int J Psychophysiol 24:39-46.

Postle BR (2006) Distraction-spanning sustained activity during delayed recognition of locations. NeuroImage 30:950-962.

Postle BR, D'Esposito M (1999) "What"-then-"where" in visual working memory: an event-related fMRI study. J Cogn Neurosci 11:585-597. 
Postle BR, Stern CE, Rosen BR, Corkin S (2000) An fMRI investigation of cortical contributions to spatial and nonspatial visual working memory. NeuroImage 11:409-423.

Postle BR, Druzgal TJ, D’Esposito M (2003) Seeking the neural substrates of visual working memory storage. Cortex 39:927-946.

Postle BR, Awh E, Jonides J, Smith EE, D'Esposito M (2004) The where and how of attention-based rehearsal in spatial working memory. Brain Res Cogn Brain Res 20:194-205.

Ranganath C, D'Esposito M (2005) Directing the mind's eye: prefrontal, inferior and medial temporal mechanisms for visual working memory. Curr Opin Neurobiol 15:175-182.

Ranganath C, Cohen MX, Dam C, D’Esposito M (2004a) Inferior temporal, prefrontal, and hippocampal contributions to visual working memory maintenance and associative memory retrieval. J Neurosci 24:3917-3925.

Ranganath C, DeGutis J, D’Esposito M (2004b) Category-specific modulation of inferior temporal activity during working memory encoding and maintenance. Brain Res Cogn Brain Res 20:37-45.

Salinas E, Sejnowski TJ (2001) Correlated neuronal activity and the flow of neural information. Nat Rev Neurosci 2:539-550.
Tallon-Baudry C, Bertrand O, Peronnet F, Pernier J (1998) Induced gamma-band activity during the delay of a visual short-term memory task in humans. J Neurosci 18:4244-4254.

Thut G, Nietzel A, Brandt SA, Pascual-Leone A (2006) $\alpha$-Band electroencephalographic activity over occipital cortex indexes visuospatial attention bias and predicts visual target detection. J Neurosci 26:9494-9502.

Troje NF, Bülthoff $\mathrm{HH}$ (1996) Face recognition under varying poses: the role of texture and shape. Vision Res 36:1761-1771.

Tuladhar AM, ter Huurne N, Schoffelen JM, Maris E, Oostenveld R, Jensen O (2007) Parieto-occipital sources account for the increase in alpha activity with working memory load. Hum Brain Mapp, in press.

von Stein A, Chiang C, Konig P (2000) Top-down processing mediated by interareal synchronization. Proc Natl Acad Sci USA 97:14748-14753.

Worden MS, Foxe JJ, Wang N, Simpson GV (2000) Anticipatory biasing of visuospatial attention indexed by retinotopically specific $\alpha$-band electroencephalography increases over occipital cortex. J Neurosci 20:RC63(1-6).

Zarahn E, Aguirre GK, D’Esposito M (1999) Temporal isolation of the neural correlates of spatial mnemonic processing with fMRI. Brain Res Cogn Brain Res 7:255-268. 